\title{
Sound movement detection deficit due to a brainstem lesion
}

\author{
T D Griffiths, D Bates, A Rees, C Witton, A Gholkar, G G R Green
}

\begin{abstract}
Auditory psychophysical testing was carried out on a patient with a central pontine lesion involving the trapezoid body, who presented with a deficit in sound localisation and sound movement detection. A deficit in the analysis of time and intensity differences between the ears was found, which would explain the deficit in detection of sound movement. The impaired detection of sound movement, due to a lesion interfering with convergence of auditory information at the superior olive, suggests this structure to be critical for human sound movement analysis.
\end{abstract}

\section{$(7$ Neurol Neurosurg Psychiatry 1997;62:522-526)}

Department of

Physiological Sciences, Newcastle University Medical School, Newcastle upon Tyne, NE2 4HH, UK

T D Griffiths

A Rees

$C$ Witton

G G R Green

Department of Clinical Neuroscience,

Newcastle University

Medical School,

Newcastle upon Tyne, NE2 4HH, UK

T D Griffiths

D Bates

Wellcome Department of Cognitive

Neurology, Institute of

Neurology, 12 Queen

Square, London,

WC1N 3BG, UK

T D Griffiths

Neuroradiology

Department,

Newcastle General

Hospital, Westgate Rd,

Newcastle upon Tyne,

NE4 6BE, UK

A Gholkar

Correspondence to:

Dr T D Griffiths,

Department of Physiological

Sciences, Newcastle

University Medical School,

Newcastle upon Tyne,

NE2 4HH,UK

Received 11 June 1996 and in final revised form 21 January 1997

Accepted 23 January 1997
Keywords: sound; sound movement; brainstem; human; lesion

The analysis of movement in the auditory system requires changes in the phase and amplitude of the sounds arriving at the two ears to be compared. Both processes depend on the convergence of information from the ears. This convergence first occurs at the superior olive, where the input from the ipsilateral cochlear nucleus converges with that from the contralateral cochlear nucleus, relayed via the trapezoid body (fig 1). Convergence also occurs higher in the pathway at the level of the colliculi. Neurophysiological studies in animals have shown that the cells in the medial superior olive (MSO) respond to timing or phase differences between the ears in a linear fashion, ${ }^{12}$ whereas cells at the level of the inferior colliculus show non-linearities consistent with a selective response to sound movement. ${ }^{3}$ Selective responses to sound movement have also been shown in animals in the superior colliculus, ${ }^{4}$ primary auditory cortex, ${ }^{567}$ and in humans in the multimodal cortex. ${ }^{8}$ Lesions producing deficits in the detection of sound movement have not previously been reported in humans, although we have recently reported a patient with a right hemispheric stroke and a deficit in the detection of phase and amplitude changes simulating motion. ${ }^{9}$ We report here a patient with a deficit in sound movement analysis due to a brainstem lesion involving the trapezoid body, suggesting that convergence at the level of the superior olive is needed for human sound movement analysis.

\section{Methods}

CASE REPORT

The patient presented at the age of 45 with recent hearing symptoms. She described difficulty with making out some speech sounds, particularly in crowded rooms, and a difficulty with sound localisation such that she had become unable to detect which of three well separated telephones in her office was ringing. The patient also had difficulty with the perception of moving sounds. For example, she was unable to detect which way a train was travelling when she was standing on a platform, on the basis of sound alone. She had a history of more than 10 years of right sided headaches and more recent right sided tinnitus, in addition to episodes of rotatory vertigo. Neurological examination was remarkable only for the presence of nystagmus on right lateral gaze.

Brain MRI with gadolinium showed an enhancing lesion in the central pons (fig 2), between the dorsal pontine tegmentum and ventral pons and extending rostrally, but not as far as the midbrain. Angiography of the posterior circulation was normal; the lesion was thought to be a capillary vascular malformation.

\section{PSYCHOPHYSICAL TESTING}

Auditory testing was carried out with computer generated stimuli presented over headphones in a sound proofed room. The tests with modulated sounds used a two alternative forced choice procedure, with a pure tone reference interval with at least 10 trials per point and at least five stimulus levels.

\section{Results}

PSYCHOPHYSICAL ASSESSMENT

Pure tone and fixed interaural phase difference detection

Pure tone thresholds disclosed a low frequency sensorineural hearing loss of $35 \mathrm{~dB}$ on the left and $25 \mathrm{~dB}$ on the right at $500 \mathrm{~Hz}$ (fig 3). These losses were corrected for in the other 


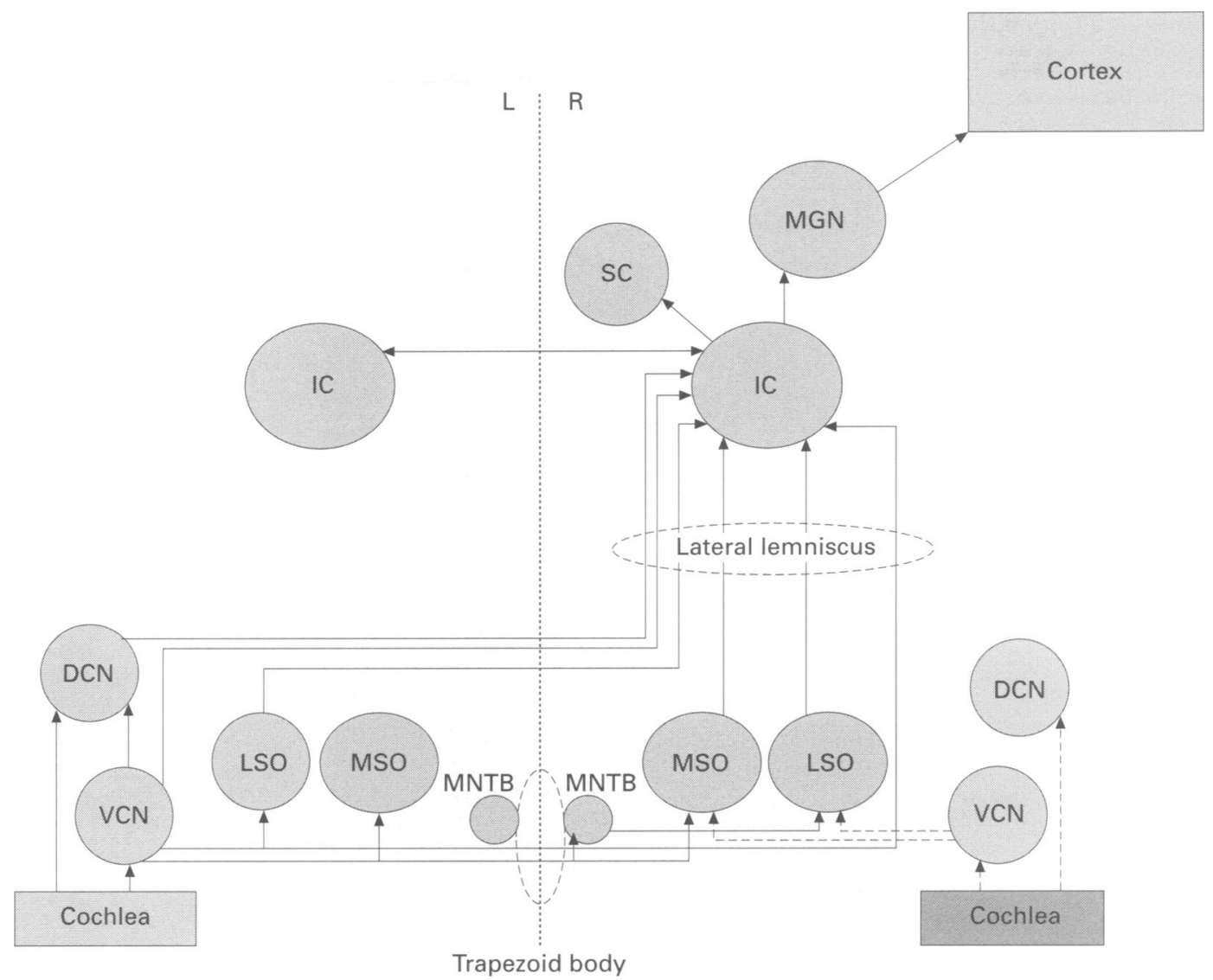

Figure 1 Simplified diagram of the auditory pathway showing the convergence of the input from both ears at the right sided brainstem auditory nucleii. This is based primarily on pathways described for the cat and other mammals ${ }^{14}$ although anatomical differences between species (including humans) are described ${ }^{16}$ ( $7 \mathrm{~K}$ Moore, personal communication). In particular, there is debate about the relative importance of the pathway via the medial nucleus of the trapezoid body (MNTB) and lateral superior olive (LSO) in humans. All connections would be reflected in the midline. The medial superior olive (MSO) is shown to be the first point at which binaural convergence occurs via the trapezoid body. Convergence also occurs at higher levels, including the level of the nucleus of the lateral lemniscus (not shown) and inferior colliculus (IC). The diagram shows a ventral pathway, via the ventral cochlear nucleus (VCN), trapezoid body, and $M S O$, thought to be important in animals for the analysis of spatial information. A dorsal pathway, via the dorsal cochlear nucleus (DCN) and IC, is also shown, which is involved in animals in the analysis of complex waveforms. MGB $=$ medial geniculate body, $S C=$ superior colliculus.

psychophysical tests by using sensation levels to match the stimulus at each ear, and additionally checking that the binaurally presented $500 \mathrm{~Hz}$ tones used in the other tests produced a midline sound image. The threshold for detection of a fixed phase difference between the ears at $500 \mathrm{~Hz}$ was $2 \cdot 25^{\circ}$ for a sound which seemed to move to the right and $3.45^{\circ}$ for a sound which seemed to move to the left, which are within normal limits. ${ }^{10}$ However, the patient found the task difficult and needed repeated trials to achieve these normal values.

Interaural phase modulation detection (sinusoidal and $\operatorname{ramp}$ )

Detection of changes in the phase or timing between the ears is used at low frequency to detect sound movement. Figure 4 shows the psychophysical function for detection of a sound in which the phase is constantly changed between the ears: sinusoidal interaural phase modulation (IPM). The stimulus is perceived as a sound which moves from side to side by normal observers, and was used as a more exacting test of the analysis of differences in phase between the ears. The patient was able to perceive this stimulus as sound movement, and had a detection threshold of $24^{\circ}$ (by Weibull analysis) compared with a mean of $18^{\circ}$ for untrained controls. A further test was carried out to check whether the patient was using phase differences between the ears, or was simply detecting the frequency modulation produced by the phase changes considered at either ear alone. This was done by playing the same phase modulated sound into the right or the left ear without a tone in the opposite ear for comparison. The patient performed at chance level on either side up to a modulation depth of $100^{\circ}$, in the same way as normal listeners, confirming that she was using phase differences between the ears to detect the IPM stimulus.

Further testing was carried out with another $500 \mathrm{~Hz}$ stimulus which contained a phase ramp, such that the phase advanced in one ear as it was delayed in the other ear. This stimulus contains identical phase changes to a sound moving in an arc around the head in one direction, and was used as a more "physiological" stimulus than one moving from side to side, in which repetition of apparent sound source positions could provide an extra cue. The patient was unable to perceive sound movement during presentation of such a ramp over $300 \mathrm{~ms}$ or $500 \mathrm{~ms}$ in either direction, even when the magnitude of the phase change was equivalent to $180^{\circ}$. Figure 5 shows the perfor- 
Figure $2 \quad$ T1 weighted gadolinium enhanced MRI. (A) Transverse section at level of the midpons showing the central location of the lesion. (B) Midsagittal section showing lesion to extend rostrally to a point below the midbrain.
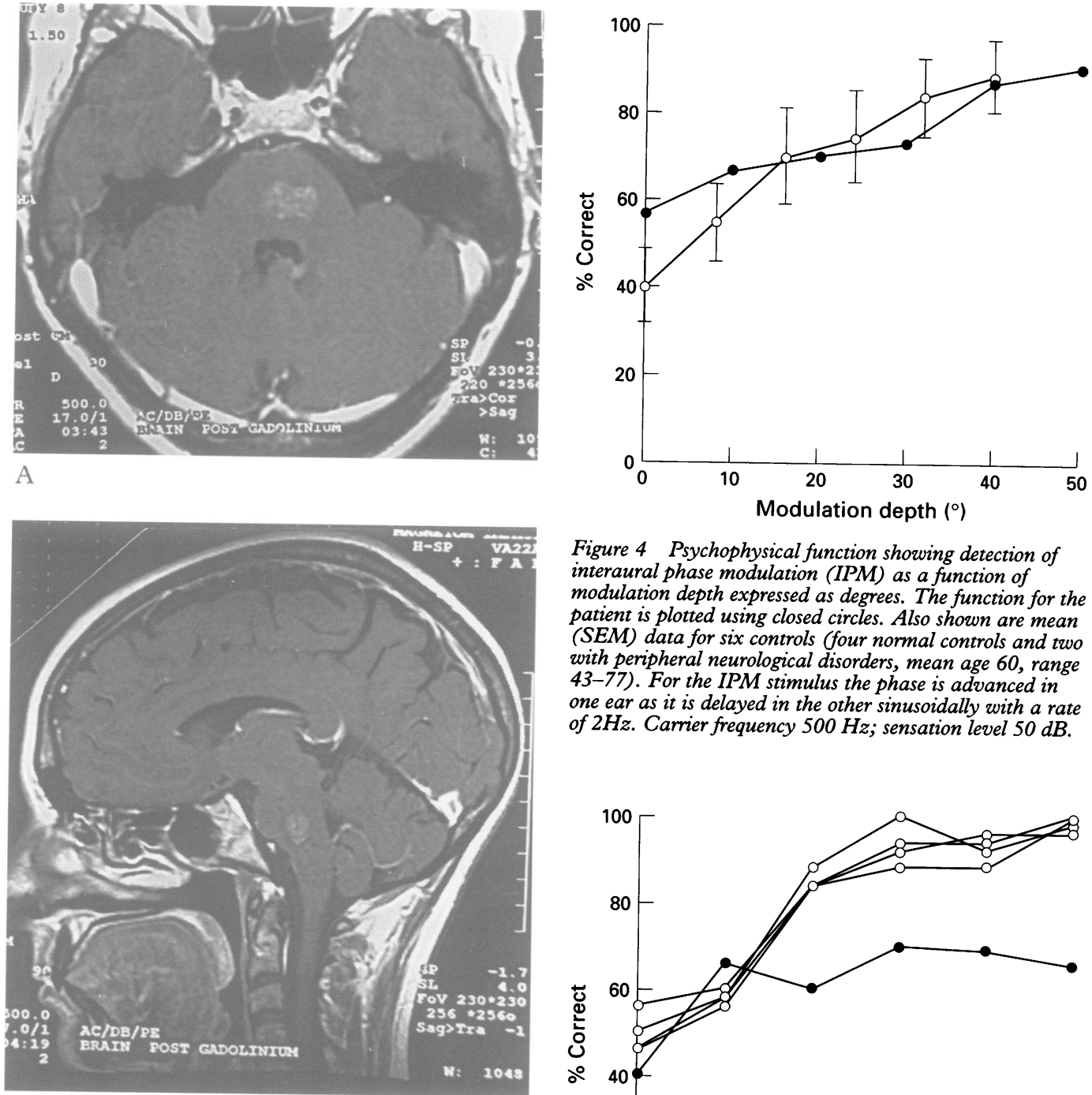

B

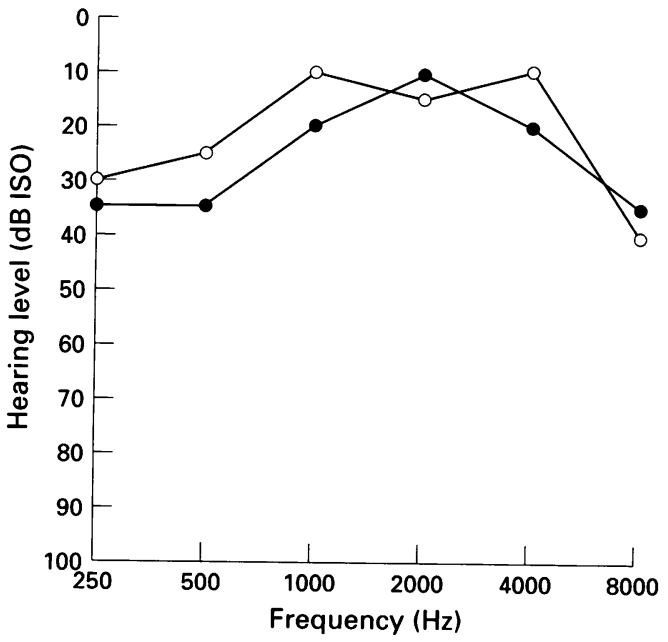

Figure 3 Pure tone audiogram of patient. Hearing levels shown as $\bigcirc$ (right) and $\bigcirc$ (left).

mance of the patient compared with normal controls for the detection of the $500 \mathrm{~ms}$ ramp.

Interaural amplitude modulation detection

Interaural amplitude modulation (IAM), which is a cue for the analysis of sound movement which is not restricted to low frequencies like phase cues, was also examined, at two car-
Figure 4 Psychophysical function showing detection of modulation depth expressed as degrees. The function for the patient is plotted using closed circles. Also shown are mean with peripheral neurological disorders, mean age 60, range 43-77). For the IPM stimulus the phase is advanced in one ear as it is delayed in the other sinusoidally with a rate of $2 \mathrm{~Hz}$. Carrier frequency $500 \mathrm{~Hz}$; sensation level $50 \mathrm{~dB}$.

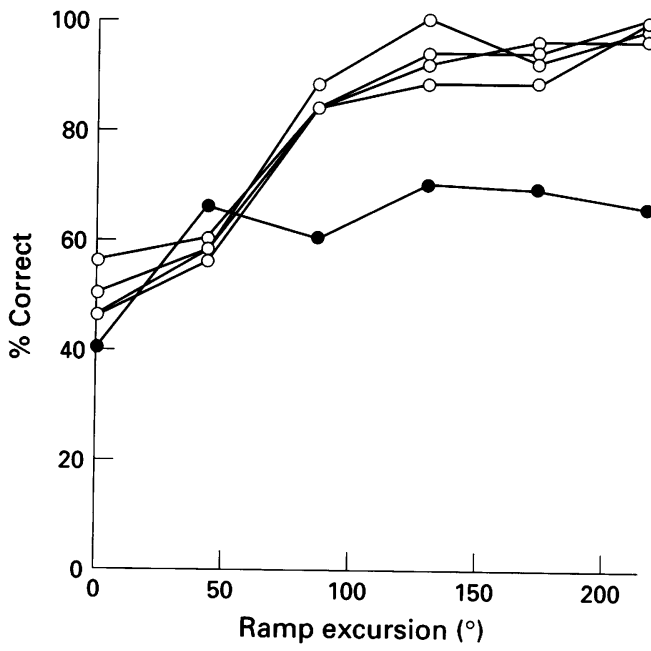

Figure 5 Psychophysical function showing detection of a $500 \mathrm{~ms}$ interaural phase ramp, simulating motion in an arc around the head, plotted as a function of phase change in degrees. The patient (O) was unable to detect any stimulus as movement, whereas the four control subject curves (O; mean age 35, range 22-45) show a threshold phase shift of $43^{\circ}$ for movement detection. The task was found easy by control subjects. Carrier frequency $500 \mathrm{~Hz}$.

rier rates of $500 \mathrm{~Hz}$ and $3 \mathrm{kHz}$. This stimulus is one in which the amplitude in one ear is increased as it is decreased in the other ear in a sinusoidal fashion, giving a perception of sound movement from side to side as in the IPM stimulus. The threshold modulation depth was $30 \%$ at both carrier rates, which is high (compared with less than $10 \%$ in three untrained normal controls aged over 50 ).

Matched frequency and amplitude modulated control stimuli

Control stimuli were used in which the frequency changes for the IPM condition and the amplitude changes for the IAM condition were identical at the two ears. These condiinteraural phase modulation (IPM) as a function of (SEM) data for six controls (four normal controls and two 
tions were used to check that the deficit in detection of differences in the waveform between the ears was specific, and not due to a more general deficit in detecting changes in sound over time. The threshold modulation depths for each condition were normal at less than $2 \mathrm{~Hz}$ for the frequency condition and less than $15 \%$ for the amplitude condition $(2 \mathrm{~Hz}$ modulation rate and $500 \mathrm{~Hz}$ carrier).

\section{Discussion}

PSYCHOPHYSICAL DEFICIT

The psychophysical assessment showed a mild asymmetric hearing loss at $500 \mathrm{~Hz}$. The degree of loss and degree of asymmetry are at a level which is lower than that at which deficits in interaural timing detection have been shown, ${ }^{11}$ although this has not been investigated for the degree of hearing loss shown by this patient. A contribution from the hearing loss and asymmetry to the deficit is still possible, however. There was a mild impairment in the detection of fixed timing differences between the ears shown by the difficulty with fixed phase difference detection. Such diffculty would contribute to the sound localisation deficit, although we were surprised not to find a more pronounced deficit in view of the symptoms described. More strikingly, deficits in the detection of varying timing differences between the ears were brought out by the ramp but not the sinusoidal interaural phase stimulus. The ramp stimulus may be more difficult to detect because in each presentation a phase transition occurs in one direction only, whereas with the sinusoidal phase modulated stimulus a phase transition occurs in both directions. This difference in the stimuli might provide an amplification of any subtle fixed phase detection deficit when a moving stimulus is used. Alternatively, the patient may have a deficit in the detection of non-linearities related to sound movement which would not be predicted by fixed spatial performance. The patient also has a deficit in the detection of varying amplitude differences between the ears shown by the impaired detection of sinusoidal amplitude differences. Both phase and amplitude deficits would contribute to the sound movement detection deficit. The normal performance in detection of sinusoidal IPM, and of binaural frequency and amplitude modulation, argue against a non-specific deficit in psychophysical performance, or general deficit in the analysis of auditory timing information.

\section{ANATOMICAL AND PHYSIOLOGICAL BASIS OF} DEFICIT

We suggest that the deficit in detection of sound movement in this patient is due to the lesion involving the trapezoid body, the tract which carries crossed auditory information from one side to allow convergence with the auditory information from the other side at the level of the superior olive (fig 1). The lateral ascending auditory pathway in the lateral lemniscus, and higher level convergence in the pathway, including at the level of the inferior and superior colliculi, are spared by the lesion.
This would provide a mechanism for preservation of fixed lateralisation, as would any residual function in the trapezoid body. Lesion studies in the cat show impaired auditory localisation due to trapezoid body lesions, ${ }^{12}{ }^{13}$ with only small increases in spatial discrimination ability for discrete lesions confined to the trapezoid body.

Convergence at the level of the colliculi and residual trapezoid body function might also be expected to allow preservation of detection of sound movement, a function ascribed to cells in the midbrain. ${ }^{34}$. This is not the case, and the pronounced deficit in detection of sound movement in this patient with disrupted superior olive input is in accord with the idea that coding of phase differences needs to occur at this level to allow analysis of movement. Whether specific movement analysis also occurs at the level of the superior olive is unclear; some non-linearities occur in animal recording work (MN Semple, MW Spitzer, personal communication) but not to the same extent as in the inferior colliculus, where analysis of sound movement is better established. $^{3}$

\section{Conclusion}

This patient with a lesion involving the trapezoid body, has a dissociated deficit in spatial analysis, particularly sound movement, with preservation of analysis of temporal sound features. This is in accord with the idea that spatial and temporal analysis of sound is separated early in the human auditory pathway, as in other mammals. The convergence of input from the two ears via the trapezoid body is important for the spatial pathway. Such deficits are not normally assessed in patients with brainstem lesions, and it will be of considerable interest to see if other patients with lesions involving the auditory pathway show similar deficits.

TDG is a Wellcome research training fellow and CW an MRC student.

1 Semple MN. Dynamic alteration of neural tuning to binaural cues: single unit responses to simulated auditory motion. cues: single unit responses to simulated auditory motion. Antwerp, Belgium:

2 Spitzer MW, Semple MN. Responses to time-varying interaural phase disparity in gerbil superior olive: evidence for hierarchical processing [abstract]. F Neurosci 1992; 18:149.

3 Spitzer MW, Semple MN. Responses of inferior colliculus neurons to time-varying interaural phase disparity: effects of shifting the locus of virtual motion. $\mathcal{F}$ Neurophysio 1993;69:1245-63.

4 Raushecker JP, Harris LR. Auditory and visual neurons in the cat's superior colliculus selective for the direction of apparent motion stimuli. Brain Res 1989;490:56-63.

5 Stumpf E, Toronchuk JM, Cynader MS. Neurons in cat primary auditory cortex sensitive to correlates of auditory motion in three-dimensional space. Exp Brain Res 1991; 88:158-68.

6 Ahissar M, Ahissar E, Bergman H, Vaadia E. Encoding of sound source location and movement: activity of single neurons and interactions between adjacent neurons in y auditory cortex. F Neurophysiol 1992;67 203-15.

7 Toronchuk JM, Stumpf E, Cynader MS. Auditory cortex neurons sensitive to correlates of auditory motion: underlying mechanisms. Exp Brain Res 1992;88:169-80.

8 Griffiths TD, Bench CJ, Frackowiak RSJ. Cortical areas in man selectively activated by apparent sound movement. Curr Biol 1994;4:892-5.

9 Griffiths TD, Rees A, Witton C, Shakir RA, Henning GB, Green GGR. Evidence for a sound movement centre in the human cerebral cortex. Nature 1996;383:425-7. 
10 Blauert J. Spatial hearing. The psychophysics of human sound localisation. Cambridge, MA: MIT Press, 1983.

11 Hausler R, Colburn S, Marr E. Sound localisation in sub jects with impaired hearing. Acta Otolaryngol 1983;

12 Casseday JH, Neff WD. Auditory localisation: role of the auditory pathways in brainstem of the cat. $\mathcal{F}$ Neurophysiol 1975;38:842-58.

13 Moore CN, Casseday JH, Neff WD. Sound localisation: the role of the commisural pathwaysof the auditory sys- tem of the cat. Brain Res 1974;82:13-26.

14 Irvine DRF. The auditory brainstem. A review of the structure and function of the auditory brainstem processing mechanisms. Berlin: Springer-Verlag, 1986.

15 Moore JK, Moore RY. A comparitive study of the superio olivary complex in the primate brain. Folia Primato (Basel) 1971;16:35-51.

16 Moore JK. The human brainstem auditory pathway. In: Jackier RK, Brackmann DE, eds. Neurotology. St Louis: Mosby, 1994. 